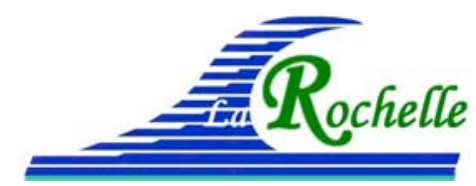

XVèmes Journées Nationales Génie Côtier - Génie Civil

La Rochelle, 29 au 31 mai 2018

DOI:10.5150/jngcgc.2018.006 ～(C) Editions Paralia CFL

disponible en ligne - http://www.paralia.fr - available online

\title{
UHAINA : A parallel high performance unstructured near-shore wave model
}

\section{Andrea Gilberto FILIPPINI ${ }^{1}$, Sébastien de BRYE ${ }^{2}$, Vincent PERRIER ${ }^{3}$, Fabien MARCHE ${ }^{4}$, Mario RICCHIUTO ${ }^{3}$, David LANNES ${ }^{2}$, Philippe BONNETON ${ }^{1}$}

1. UMR EPOC, University of Bordeaux, Allée Geoffroy Saint-Hilaire, 33615 Pessac cedex, France. andrea-gilberto.filippini@u-bordeaux.fr ; philippe.bonneton@u-bordeaux.fr

2. IMB, University of Bordeaux, 351 cours de la Libération, 33405 Talence cedex, France. sebastien.de-brye@u-bordeaux.fr ; david.lannes@math.u-bordeaux.fr

3. INRIA Bordeaux sud-Ouest, 200 av. de la vieille tour, 33405 Talence cedex, France. vincent.perrier@inria.fr ; mario.ricchiuto@inria.fr

4. IMAG, University of Montpellier, Place Eugène Bataillon, 34090 Montpellier, France.

Fabien.Marche@umontpellier.fr

\begin{abstract}
:
UHAINA is a new phase-resolving free surface wave model for coastal engineering problems. It is based on the most advanced and recent contributions in coastal modelling from the french institutes EPOC, IMAG, IMB, and INRIA BSO. It solves a non-classical version of the depth-integrated fully-nonlinear and weakly-dispersive equations of Green-Naghdi, which allows an efficient numerical implementation. UHAINA relies on libraries developed at the INRIA BSO center, such as AeroSol for its hydrodynamic core, and PaMPA and SCOTCH to handle data management for distributed memory parallel computation. The use of these libraries, in particular AeroSol, offers a wide range of possibilities including arbitrary high-order finite element discretizations, hybrid meshes (structured and unstructured), as well as an advanced programming environment specially designed by the purpose of performance and HPC. These properties will lead in the coming years to the release of a new efficient and robust open source wave modelling platform, available for a large community of users and very suitable for practical coastal applications.
\end{abstract}

Keywords: Green Naghdi equations, Phase-resolving wave model, Wave breaking, Discontinuous Finite Element, Unstructured meshes. 


\section{Thème 1 - Hydrodynamique côtière}

\section{Introduction}

For several decades the population density in coastal areas has significantly increased. This trend is expected to continue in the next decades. Consequently, extreme ocean events, such as tsunamis or storm waves, have increasing damaging consequences. This makes risk assessment a crucial element for the safe development of these communities. In this context, it is essential to dispose of robust and yet efficient models for predicting extreme events involving the propagation of waves in the near shore, and their impact on the coast. Over the past years, strong efforts have been made by the French laboratories and research centers EPOC, IMAG, IMB, and INRIA BSO to advance the state of wave modelling and flooding simulations for coastal engineering applications. These efforts have recently focused on developing and validating the newly developed non-hydrostatic wave-flow model UHAINA. This model will integrate the collective know how on non-linear wave modelling, high order numerical discretizations, and high performance object oriented implementation developed in the last decade. Typical applications of UHAINA will be the study of the propagation and transformation of waves in the surf and swash zones, such as wave shoaling, dispersion and breaking together with coastal flooding and structure overtopping.

In recent years, Boussinesq wave models have become a useful tool for modeling surface wave transformation from deep water to the swash zone. Great improvements have been obtained in the derivation and mathematical understanding of particular asymptotic models able to describe the behaviour of the solution in some physical specific regimes; a recent review on different existing models is given in LANNES \& BONNETON (2009). In particular, great efforts have been focused on improving the range of model applicability with respect to classical restrictions to both weak dispersion and weak nonlinearity. The use of the so-called fully-nonlinear formulation of GREEN \& NAGHDI (1978) eliminates the restriction to weak nonlinearity, enhancing the models capabilities in the surf and swash zones, where the wave breaking point is attended in conditions of increased nonlinearity. For these reasons, the GreenNaghdi equations have gained a lot of attention in the recent past. UHAINA uses a nonclassical variant of this model with improved linear dispersion properties and which allows a faster solution procedure (LANNES \& MARCHE, 2015).

Phase-resolved modelling, based on Boussinesq-type equations and in conjunction with suitable numerical techniques, has emerged as a mature discipline and generated the most widely employed predictive tools in coastal engineering and in morphodynamics, as e.g. FUNWAVE (KIRBY et al., 1998) or COULWAVE (LYNETT \& LIU, 2002). UHAINA fits into this category of models, embedding the most recent research progresses in coastal wave modelling, making them accessible to anyone. To do so, the platform relies on libraries developed at Inria, such as the AeroSol finite element library (for its hydrodynamic core), and the libraries PaMPA and SCOTCH to handle transparently for the model developer data management in distributed memory parallel 


\section{XVèmes Journées Nationales Génie Côtier - Génie Civil \\ La Rochelle, 29 au 31 mai 2018}

computations. The use of these libraries, and in particular AeroSol, offers a wide range of possibilities including both continuous and discontinuous High-Order finite element discretizations, hybrid meshes (structured and unstructured), as well as a code design driven by performance purposes (HPC) using advanced $\mathrm{C}++$ programming techniques. In what follows, Section 2 summarises the essential model, as well as numerical and computational aspects featuring in UHAINA. In Section 3, a few application examples are then presented, reflecting both capabilities and performances. Finally, Section 4 concludes the paper with some closing remarks.

\section{Modelling framework}

\subsection{Physical model}

Modeling nonlinear coastal wave processes, such as inundation, wave runup, bore propagation, tsunami propagation, and infragravity waves, requires efficient and accurate computing of the evolution of highly nonlinear and dispersive surface wave fields in complex coastal environments. UHAINA relies on a phase-resolving approach, based on the fully-nonlinear and weakly-dispersive Boussinesq wave model of GREEN \& NAGHDI (1978). This system of equations shares the same linear dispersion properties of the original Boussinesq model of PEREGRINE (1967), however an equivalent model with improved dispersion properties has been proposed in (CHAZEL et al., 2011), by the introduction of a tuning parameter $\alpha$ multiplying some dispersive terms. The new formulation of the governing equations, proposed by BONNETON et al. (2011), allows the system of governing equations to be rewritten as follows:

$\partial_{t} \zeta+\nabla \cdot(h \boldsymbol{u})=0$

$\partial_{t}(h \boldsymbol{u})+\nabla \cdot(h \boldsymbol{u} \otimes \boldsymbol{u})+g h \nabla \zeta=\boldsymbol{\phi}$

$(I+\alpha T)(\phi)-T(g h \nabla \zeta)+h Q(\boldsymbol{u})=0$

having used $\zeta$ to indicate the free surface elevation, $h$ for the total water depth, $\boldsymbol{u}$ for the velocity vector, and where $\phi$ accounts for the non-hydrostatic effects, $I$ is the identity matrix, while $T$ and $Q$ are operators containing high-order derivatives in space (for more details on their definitions please refer to the cited works). A two steps solution procedure is applied to the system (1)-(3), as described in FILIPPINI et al. (2016). It consists in: an elliptic phase (3) in which the source term $\phi$ is computed by inverting the coercive operator associated to the dispersive effects; an hyperbolic phase in which the flow variables are evolved by solving the Shallow Water equations (1)-(2), with all nonhydrostatic effects accounted for by the source $\phi$ computed in the elliptic phase. The main advantage of this formulation is the presence of the operator $(I+\alpha T)$, which makes the model robust with respect to high frequency perturbations, an interesting property for numerical computations. However, the inversion of the $(I+\alpha T)$ matrix, for the solution of the elliptic phase, is the most computationally demanding part of the whole solution process. This is due to the fact that, firstly, $(I+\alpha T)$ is a matricial second order 


\section{Thème 1 - Hydrodynamique côtière}

differential operator acting on two-dimensional vectors and this structure entails a coupling of the time evolutions of the two components of hu through (2). Secondly, $T(h(x, t))$ is a time-dependent operator, through the dependence on $h$ : the corresponding matrices have, thus, to be assembled at each time step or sub-steps. In order to overcome these drawbacks without loosing the benefits of the formulation (1)-(3), UHAINA exploits new very promising non-classical models derived by LANNES \& MARCHE (2015). While keeping the same asymptotic $\mathrm{O}\left(\mu^{2}\right)$ order (being $\mu=\left(h_{0} / \lambda\right)^{2}$ the dispersion parameter, with $h_{0}$ the reference water depth and $\lambda$ the typical wave length), and linear properties of the original model, LANNES \& MARCHE (2015) have shown that it is possible to rewrite the elliptic equation (3) in a way such that the new operator to invert to be either block diagonal or block diagonal and time-independent, leading to considerable improvement in terms of computational time, since it allows to perform the corresponding matrix assembling and factorization in a pre-processing step. For the representations of dissipative wave-breaking events, UHAINA exploits the hybrid strategy of TISSIER et al. (2012), KAZOLEA et al. (2014) and DURAN \& MARCHE (2017), by locally reverting to the Shallow Water equations to model energy dissipation in breaking regions.

\subsection{Numerical discretization}

From the numerical point of view the Green-Naghdi equations have been discretized using different numerical techniques including Finite Differences (FD), Finite Elements (FE) and Finite Volumes (FV) approaches. The major challenges that need to be dealt with are the approximation of the complex higher order derivative terms, in respect of the accuracy requirements on the schemes in terms of low dispersion errors. Fully unstructured solvers, allowing for adaptive mesh refinement, have been proposed based either on a hybrid FV/FE approach (FILIPPINI et al., 2016) or on a discontinuous FE approach (DURAN \& MARCHE, 2017). Inspired by these works, UHAINA is focused on the application of a FE discretization of the governing equations. This gives a framework to naturally introduce higher order polynomial representation of the unknowns and of their derivatives.

UHAINA adopts an arbitrary high-order discontinuous FE discretization of the hyperbolic phase, exploiting the robustness and shock capturing capabilities of this approach in wave breaking regions, where the Shallow Water equations are solved, due to the hybrid breaking model used. The use of a nodal approach together with the prebalanced formulation of the hyperbolic part of the model allows to combine two important properties (DURAN \& MARCHE, 2017): firstly, an efficient quadrature free treatment for the integrals which are not involved into the equilibrium state preservation; secondly, a quadrature-based treatment with a lower computational cost, needed to exactly compute the surface and face integrals involved in the preservation of the steady states at rest. Moreover discontinuous FE approach has a compact stencil; a 


\section{XVèmes Journées Nationales Génie Côtier - Génie Civil \\ La Rochelle, 29 au 31 mai 2018}

property which makes it well-suited for parallel computing. Concerning the discretization of the elliptic phase, a second order FE approach is here used, leading for the fully coupled scheme to a phase accuracy very close to that of a fourth order FD method, as stated in the works of FILIPPINI et al. (2016) and DURAN \& MARCHE (2017).

\subsection{HPC Implementation}

High-order accuracy, computational efficiency and parallelism are among the main targets of the UHAINA platform. In order to achieve these objectives UHAINA relies on the Aerosol library, developed at the INRIA SO institute. Aerosol is a C++ library, devoted to the solution of complex CFD problems and recently adapted to deal also with hydrodynamic applications. It is a high order finite element library based on both continuous and discontinuous elements on hybrid meshes, involving triangles and quadrangles in two dimensions. More precisely, it enables the generation of finite element classes up to the fourth order polynomial approximation. The code design is driven by the purpose of performance using advance $\mathrm{C}++$ programming techniques and employs an efficient parallel implementation, which allows high performance computing on massively parallel architectures. Aerosol depends on the PaMPA library for memory handling, for mesh partitioning, and for abstracting the MPI layer, and it is also linked with external linear solvers (e.g. BLAS, PETSc 4 and MUMPS 5 ).

At present, only the hyperbolic part of the model is operational, while the development of the non-hydrostatic part of the model is currently underway.

\section{Preliminary results}

\subsection{Convergence test}

Firstly, we report a convergence study performed on an academic test, which allows us to asses the theoretical order of accuracy of the implemented scheme.

The test proposed consists in propagating a subcritical flow over a submerged bump described by the function $z=0.5(2 \pi)^{-1 / 2} \exp \left(-0.5(x-10)^{2}\right)$. The free surface elevation is initially set as constant at $\eta=2[\mathrm{~m}]$ and a constant discharge of $q=4.42$ $\left[\mathrm{m}^{2} / \mathrm{s}\right]$ is injected from the left boundary of the domain, while an open boundary is simulated on the right. The result of the simulation is shown in Figure 1 (left). The test is performed using the nonlinear shallow water model, for which an analytical solution to this problem exists (GOUTAL \& MAUREL, 1998). This allows us to perform a grid convergence of the error using different order of polynomial approximation in our numerical scheme. The test case is performed on a set of four meshes successively dividing the space step by two up to $d x=0.125[\mathrm{~m}]$, while keeping the time step small enough to ensure that the leading error order is provided by the spatial discretization. 


\section{Thème 1 - Hydrodynamique côtière}

The slopes obtained from the errors, in Figure 1 (right), reveal that the convergence rates of the scheme match the theoretical values for all the combinations.
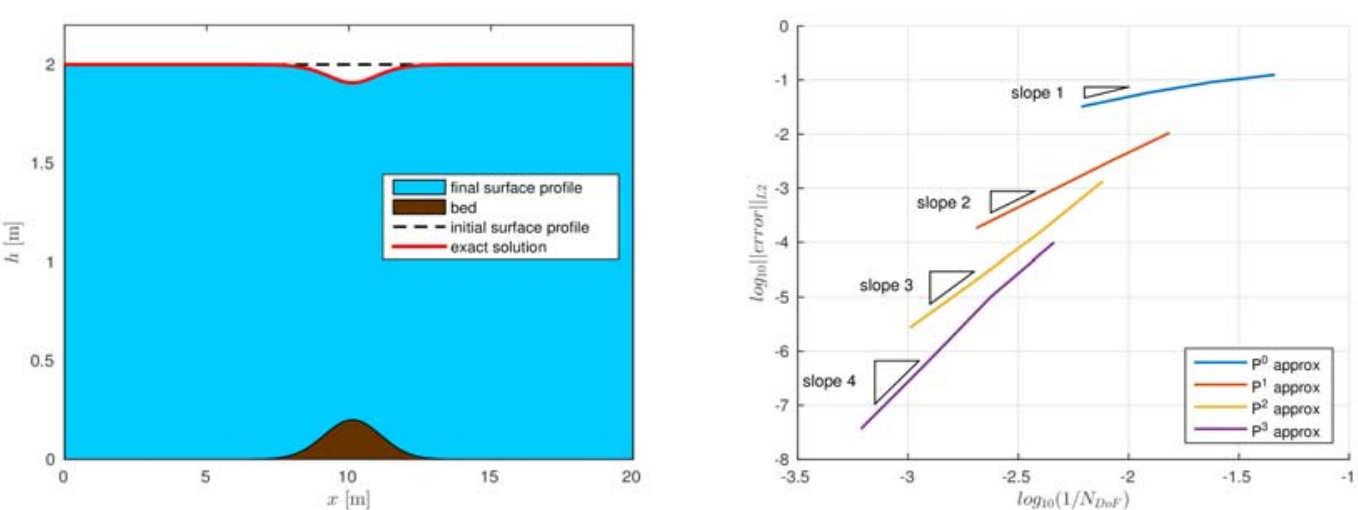

Figure 1. Left: Steady subcritical flow over a bump; illustration of the profile of water surface and bottom. Right : convergence of the $L^{2}$ norm of the error with respect to the inverse of the number of degrees of freedom, when polynomial approximations of order $P^{0}, P^{1}, P^{2}$ and $P^{3}$ are used in the scheme.

\subsection{Real case application}

Hereafter, UHAINA is applied to numerically reproduce the laboratory experiment of PARK et al. (2013). This is a very recent benchmark test for tsunami inundation of an urban waterfront. A 1:50 scale idealization of the town Seaside, Oregon was designed to observe the impact of a tsunami wave and measure the water flows which are produced around the city buildings. The scale model was installed in a rectangular basin with a wavemaker on the offshore boundary. Free surface elevation and velocity time series were measured and analyzed at 31 points along 4 transects (please refer to the cited work for the precise setup of the experiment and gauges positions).

The numerical simulation has been performed using the shallow water model. Figure 2 shows two different views of the computational domain during the simulation, one before (left) and the other just after (right) the tsunami impacting the town.

The computed free surface time series at some significant gauges (Figure 2, left), located along the central street of the city, are illustrated in Figure 3 and compared with respect to the experimental ones. The comparisons show a good agreement between the numerical and the physical model in the most of the locations inspected. However, as known, the shallow water equations, applied for the numerical simulation, underestimate the shoaling of the tsunami wave over the sloping beach. This cause the computed peak of the first incoming wave to be smaller than the experimental one, an effect which is visible in the signal registered at gauge B1 (the one situated close to the seaside). 


\section{XVèmes Journées Nationales Génie Côtier - Génie Civil \\ La Rochelle, 29 au 31 mai 2018}

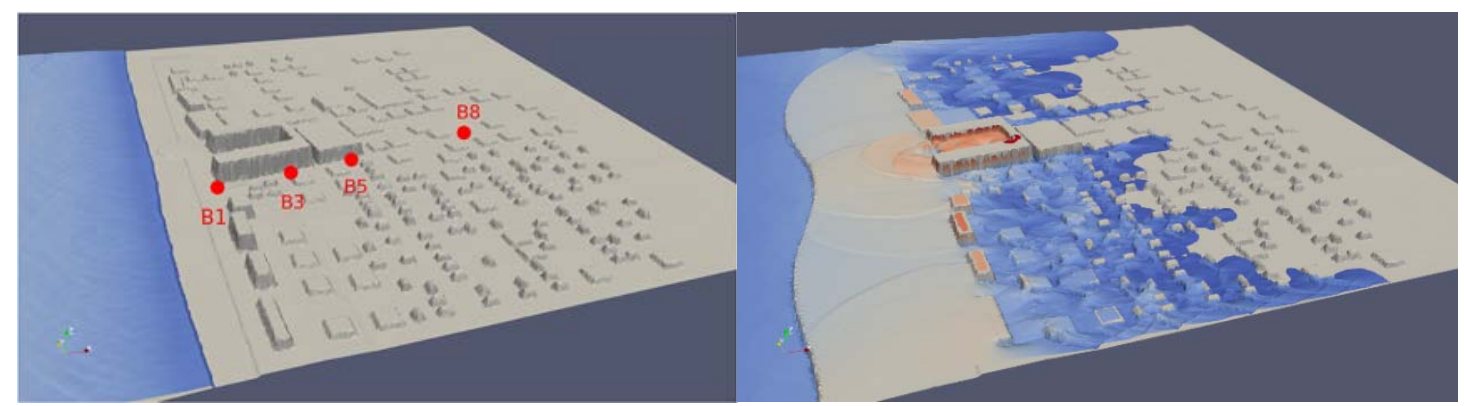

Figure 2. Three dimensional views of the Seaside numerical simulation, at two different instant of the computation: before (left) and just after (right) the tsunami arrival. Red bullets correspond to some gauges positions.
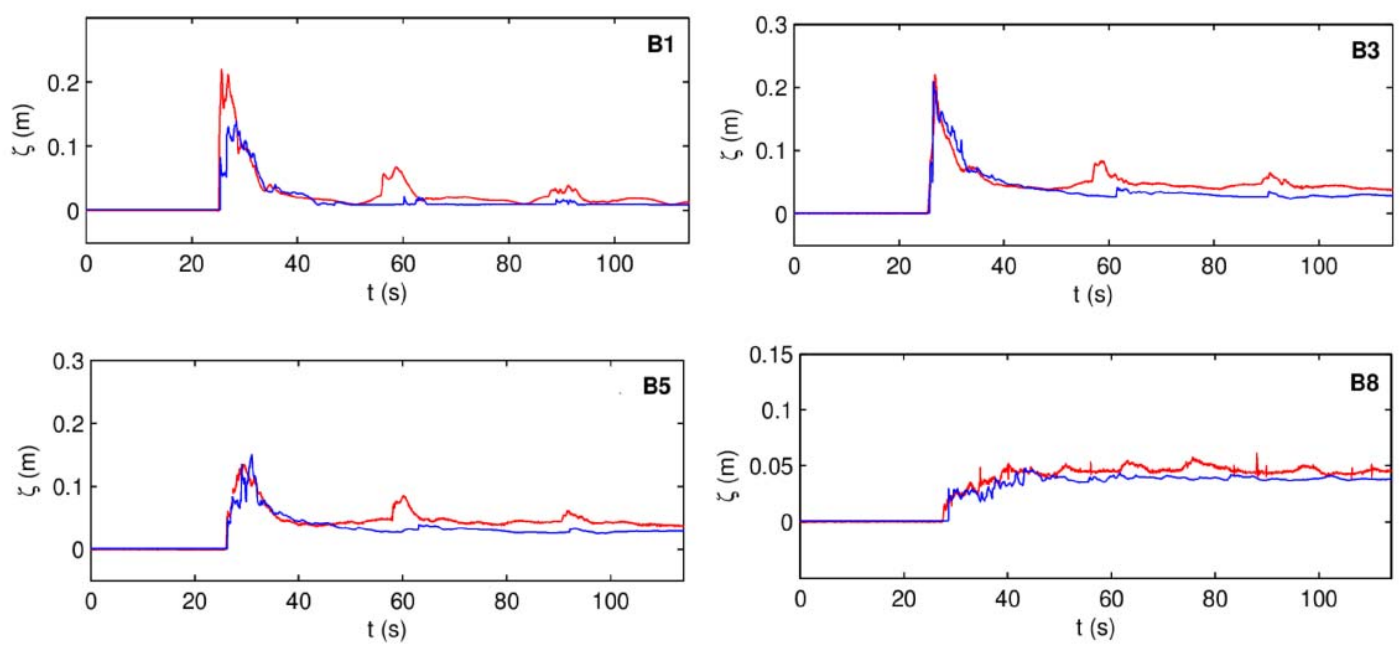

Figure 3. Time series of the free surface elevation at some gauges positions along the main street of the city, perpendicular to the sea line: blue lines indicate the results of the simulation, while red lines stay for the experimental ones.

\section{Conclusions and perspectives}

A new non-hydrostatic wave-flow modelling platform named UHAINA is presented in this work. It is devoted to the prediction of surface waves transformation processes in coastal waters, gathering the following numerical properties: it provides arbitrarily highorder discretization of a new non-classical formulation of the Green-Naghdi equations; it works on unstructured meshes; it exploits an efficient parallel implementation, allowing HPC, through the use of the Aerosol library and its dependencies.

To validate the currently operational part of the code and to demonstrate its potential, two test cases have been presented for demonstration and validation purposes, showing that an arbitrary order of accuracy of the numerical scheme is correctly obtained and that the scheme is able to correctly reproduce a realistic case of study in a complex flooding scenario. 


\section{Thème 1 - Hydrodynamique côtière}

Further developments will lead in the coming years to a favorable environment for a large community of users to perform real-time large simulations with pre- and postprocessing of the data, and will make UHAINA a new generation robust and computationally efficient phase-resolving numerical wave model very suitable for practical application studies.

\section{References}

BONNETON P., CHAZEL F., LANNES D., MARCHE F., TISSIER M. (2011). A splitting approach for the fully nonlinear and weakly dispersive Green-Naghdi model. J. Comp. Phys., Vol. 230(4), pp 1479-1498. https://doi.org/10.1016/j.jcp.2010.11.015

CHAZEL F., LANNES D., MARCHE F. (2011). Numerical simulation of strongly nonlinear and dispersive waves using a Green-Naghdi model. J. Sc. Comp., Vol. 48(1-3) pp 105-116. https://doi.org/10.1007/s10915-010-9395-9

GOUTAL N., MAUREL F. (1998) Dam-break wave simulation. Proceeding in 1st CADAM workshop

DURAN A., MARCHE F. (2017). A discontinuous Galerkin method for a new class of Green-Naghdi equations on simplicial unstructured meshes. Appl. Mat. Mod., Vol. 45, pp 840-864. https://doi.org/10.1016/j.apm.2017.01.030

FILIPPINI A.G., KAZOLEA M., RICCHIUTO M. (2016) A flexible genuinely nonlinear approach for nonlinear wave propagation, breaking and run-up. J. Comp. Phys., Vol. 310, pp 381-417. https://doi.org/10.1016/j.jcp.2016.01.027

GREEN A.E., NAGHDI P.M. (1978). A derivation of equations for wave propagation in water of variable depth. J. Fluid Mech., Vol. 78, pp 237-246. https://doi.org/10.1017/S0022112076002425

KAZOLEA M., DELIS A.I., SYNOLAKIS C. (2014). Numerical treatment of wave breaking on unstructured finite volume approximations for extended Boussinesq type equations. J. Comp. Phys., Vol. 271, pp 281-305. https://doi.org/10.1016/j.jpp.2014.01.030

KIRBY J.T., WEI G., CHEN Q., KENNEDY A., DALRYMPLE R.A. (1998) FUNWAVE 1.0 : Fully nonlinear Boussinesq wave model documentation and User's manual. CACR-98-06, University of Delaware, Newark, DE 19716, USA.

LANNES D., BONNETON P. (2009). Derivation of asymptotic two-dimensional timedependent equations for surface water wave propagation. Phys. of Fluids, Vol. 21. https://doi.org/10.1063/1.3053183

LANNES D., MARCHE F. (2015). A new class of fully nonlinear and weakly dispersive Green-Naghdi models for efficient 2D simulations, J. Comp. Phys., Vol. 282, pp 238-268. https://doi.org/10.1016/j.jpp.2014.11.016

LYNETT P.J., LIU P.L. (2002) Modeling wave generation, evolution, and interaction with depth-integrated, dispersive wave equations. COULWAVE code manual Cornell University, Ithaca, NY, USA. 


\section{XVèmes Journées Nationales Génie Côtier - Génie Civil \\ La Rochelle, 29 au 31 mai 2018}

PARK H., COX D.T., LYNETT P.J., WIEBE D.M., SHIN S. (2013) Tsunami inundation modeling in constructed environments: A physical and numerical comparison of free-surface elevation, velocity, and momentum flux. Coast. Eng., Vol. 79, pp 9-21. https://doi.org/10.1016/j.coastaleng.2013.04.002

PEREGRINE D.H. (1967). Long waves on a beach. J. Fluid Mech.,Vol. 27, pp 815-827. https://doi.org/10.1017/S0022112067002605

TISSIER M., BONNETON P., MARCHE F., CHAZEL F., LANNES D. (2012). A new approach to handle wave breaking in fully nonlinear Boussinesq models. Coast. Eng., Vol. 67, pp 54-66. https://doi.org/10.1016/j.coastaleng.2012.04.004 
Thème 1 - Hydrodynamique côtière 